\title{
Galactomannan and 1,3- $\beta$-D-Glucan Assay in Bronchoalveolar Lavage Fluid for the Diagnosis of Invasive Pulmonary Aspergillosis in Malignant and Non-malignant Patients
}

\author{
Hadir Ahmed El-Mahallawy ${ }^{1}$, Rana Mahmoud El-Gendi*(i), Doaa Mohammad Ghaith ${ }^{3}$, Iman Kamal Behiry ${ }^{3}$ (D) \\ Sohier Fathy Helal ${ }^{3}$ D \\ ${ }^{1}$ Department of Clinical Pathology, National Cancer Institute, Cairo University, Cairo, Egypt; ${ }^{2}$ Department of Clinical Pathology, \\ Abbassia Fever Hospital, Ministry of Health and Population, Cairo, Egypt; ${ }^{3}$ Department of Clinical and Chemical Pathology, \\ Faculty of Medicine, Cairo University, Cairo, Egypt
}

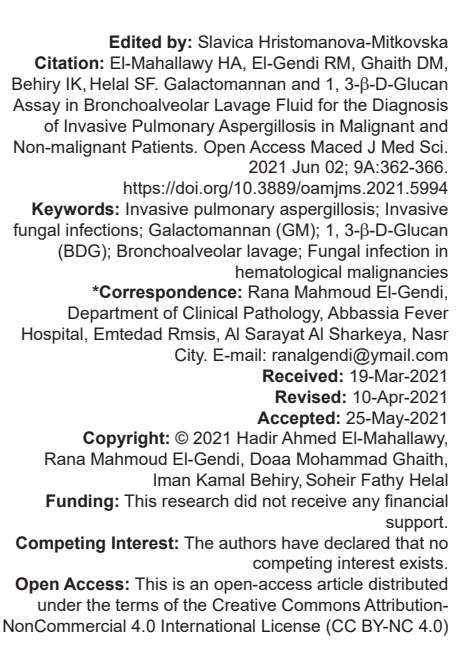

Abstract

BACKGROUND: Serum 1, 3- $\beta$-D-glucan (BDG) assay was recommended for diagnosing fungal infections.

AIM: We aimed to assess 1, 3- $\beta$-D-glucan in bronchoalveolar lavage (BAL) fluid in invasive pulmonary aspergillosis (IPA).

METHODS: Out of 104 patients clinically suspected fungal, 45 were probable, 18 possible, and 41 unlikely according to EORTC/MSG 2008 criteria. Measuring BAL BDG and galactomannan were done.

RESULTS: The sensitivity, specificity, and positive and negative predictive values (PPV and NPV) for BDG were $44 \%$, $71 \%, 62 \%$, and $54 \%$; for galactomannan $84 \%, 83 \%, 84 \%$, and $83 \%$; and $93 \%, 66 \%, 75 \%$, and $90 \%$, respectively, when combining both tests. A significant different performance of $\mathrm{GM} ; \mathrm{p}=0.0008$ was detected in patients with malignant disorders when compared to non-malignant; but not for BDG; $p=0.121$.

CONCLUSION: We can conclude that BAL-BDG is helpful if positive in a clinically suspected IFI case, but if negative cannot rule out fungal infection. Thus, combining results of BAL-GM and BAL-BDG are recommended.

\section{Introduction}

Invasive pulmonary aspergillosis (IPA) is a life-threatening disease, affecting oncology patients, critically ill patients, and those with chronic obstructive pulmonary disease (COPD) [1]. The diagnosis of IPA remains challenging. In 2016, Infectious Disease Society of America (IDSA) recommended simultaneous histopathologic and culture examination for a proven diagnosis of IPA. Serum assay of $(1,3)-\beta$-D-glucan is recommended for diagnosing invasive aspergillosis in high-risk patients, such as hematologic malignancy or allogeneic hematopoietic stem cell transplant (HSCT), but it is not specific for Aspergillus [2].

Beta-D-glucans comprise part of the polysaccharides naturally occurring in the cell walls of cereals, yeast, bacteria, and fungi [3]. The cell walls of Aspergillus contain relatively large amounts of $1,3-\beta$-Dglucan and galactomannan, which are released during logarithmic growth, and slightly later [4]. Unlike GM, BDG is widely distributed in the fungal kingdom and is found in the cell walls of many pathogenic fungi including Candida, Fusarium, Acremonium, Pneumocystis, and Histoplasma capsulatum though it is present at a lower level in Cryptococcus and virtually undetectable in the mucoraceous molds [5].

The European Organization for Research and Treatment of Cancer/Mycosis Study Group (EORTC/MSG) developed criteria for the diagnosis of invasive fungal infection, and these criteria were revised and modified later to include more recent indirect tests, such as galactomannan and beta-D- glucan [6]. In a previous study performed at National Cancer Institute, Cairo University, Aspergillus was the most common pathogen causing IFI, and the lower respiratory tract was the most common site of infection [7]. The BDG test has not been validated in BAL specimens. Thus, we aimed to assess the performance of testing BDG in BAL as a single test and combined with BAL GM for the rapid diagnosis of invasive pulmonary aspergillosis. 


\section{Methodology}

This study was a prospective study conducted in hospitals of KasrAlAiny Hospital (KA), Cairo University, and National Cancer Institute (NCl), Cairo University, during the period from August 2016 to May 2018 after approval of the research ethics committee. Patients included in the study were clinically suspected of fungal infections of the lower respiratory tract. Inclusion criteria were mainly fever and respiratory symptoms not responding to empirical and/or therapeutic antibacterial therapy for at least $96 \mathrm{~h}$; together with one of the following predisposing factors neutropenia less than $500 / \mathrm{mm}^{3}$ more than 7 days, in malignant cases, corticosteroid therapy more than 10 days, patients on a mechanical ventilator or other respiratory devices and radiographic features suggesting invasive fungal infection. Exclusion criteria included infectious episode lasting less than $96 \mathrm{~h}$ acute respiratory infections responding to antibiotics and contaminated culture plates.

\section{EORTC categories}

The patients were classified into proven, probable, possible, and unlikely, according to the revised definition of IFI from the European Organization for Research and Treatment of Cancer-Invasive Fungal Infections Cooperative Group and the National Institute of Allergy and Infectious Diseases Mycoses Study Group (EORTC/MSG). Proven invasive fungal infection required only that a fungus be detected by histological analysis or culture of a specimen taken from a sterile site [2]. Probable invasive fungal infections hinged on three elements, namely, a host factor that identified the patients at risk, such as neutropenia $<500 / \mathrm{mm}^{3}$ for more than 10 days, receipt of allogeneic stem cell transplant, prolonged use of corticosteroids, T-cell immunosuppressors during the past 90 days, and inherited severe immunodeficiency. Clinical criteria for a probable case were a CT with one of the following findings: Dense well-circumscribed lesion(s) \pm halo sign, air crescent sign, or cavitation; together with mycological evidence that encompassed culture, microscopic analysis, and a positive indirect test, such as galactomannan. Possible invasive fungal infections have the same criteria of probable except mycological findings.

\section{Laboratory workup}

BAL samples were collected from all patients and aliquoted into three parts; the first part was examined microscopically for fungal elements, the second part was cultured on Sabouraud's dextrose agar (SDA) plates; one incubated at $25^{\circ} \mathrm{C}$ and the other was incubated at $45^{\circ} \mathrm{C}$ and all plates were examined daily for fungal growth over a period of 2 weeks, the third part was tested for 1, 3-beta-D-glucan level using Fungitell assay (Associates of Cape Cod, Inc.), and galactomannan, Platelia, Bio-Rad, 92430 Marnes-laCoquette, France.

For (1-3)- $\beta$-D-glucan, values <60 pg./mL were interpreted as negative results, values $>80 \mathrm{pg} . / \mathrm{mL}$ are interpreted as positive and intermediate values from 60 to $80 \mathrm{pg} . / \mathrm{mL}$ suggest a possible fungal infection. For galactomannan, samples with an optic density index (ODI) $<1$ were considered to be negative for galactomannan antigen, and samples with an index $\geq 1$ were considered to be positive for galactomannan antigen. Both tests were performed according to the manufacturers' instructions.

Statistical analysis was done using $\mathrm{IBM}^{\odot}$ SPSS $^{\odot}$ Statistics version 22 (IBM ${ }^{\odot}$ Corp., Armonk, NY, USA). Numerical data were expressed as mean and standard deviation or median and range as appropriate. Qualitative data were expressed as frequency and percentage. Chi-square test or Fisher's exact test was used to compare qualitative variables, while MannWhitney test (non-parametric t-test) was used for not normally distributed quantitative data. Evaluation of the diagnostic tests was done by calculating sensitivity, specificity, positive predictive values (PPV), negative predictive value (NPV), and total accuracy according to EORTC/MSG definition probable against unlikely.

\section{Results}

During 2 years duration, 233 patients were clinically suspected to have IFI referred to microbiology laboratory by physicians from different Cairo University hospitals. Of the 233 patients, 104 fulfilled our criteria; 29 cases from $\mathrm{KA}$ and 75 from $\mathrm{NCl}$. Their age ranged from 18 to 91 with a median of 49.5 years. Out of 104 patients, 57 patients were male and 47 were female, with a male-to-female ratio of (1.21). Sixtyfour patients came from rural areas and 40 came from urban areas. Regarding hospitalization, 90 patients were hospitalized, among which 30 were admitted to intensive care units. The majority of our patients (79/104) had malignant diseases, 48 patients had hematological malignancies (acute myeloid leukemia, acute lymphocytic leukemia, non-Hodgkin lymphoma, and chronic lymphocytic leukemia) and 31 had solid organ malignancies (bladder cancer, uterus/ovarian carcinoma, lung cancer, GIT malignancy, schwannoma, sarcoma, and mesothelioma) while 25 had nonmalignant diseases, such as COPD and bronchiectasis. CT findings suspected fungal infections in 63 patients.

According to EORTC revised criteria (2008) [6], patients were classified into 45 probable cases of IFI, 18 possible cases, and 41 patients negative for IFI. No proven cases were detected in our study as no tissue biopsy was obtained. EORTC categories were differently distributed among malignant and nonmalignant patients, as shown in Figure 1. 


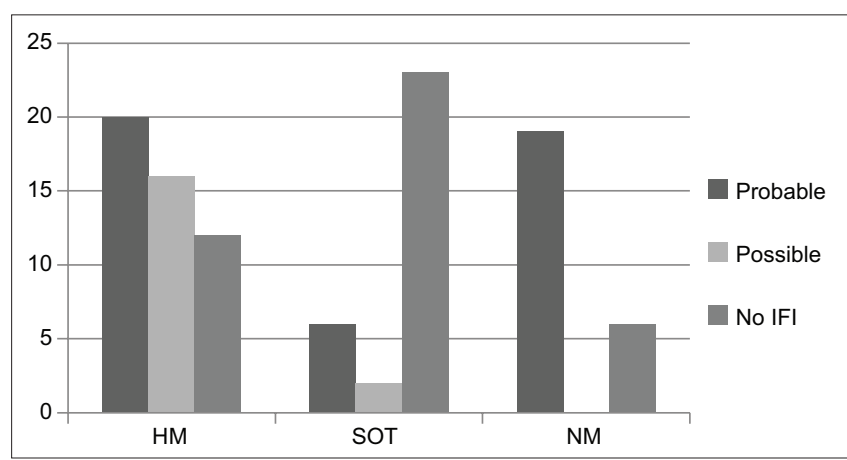

Figure 1: Distribution of probable, possible, and unlikely cases among malignant and non-malignant patients in 104 clinically suspected IF patients. Hematological malignancies: 20 probable, 16 possible, and 12 no IFI. Solid organ malignancies: 6 probable, 2 possible, and 23 no IFI. Non-malignant: 19 probable, 0 possible, and 6 no IFI

\section{Laboratory results}

Laboratory test results were as follows, direct microscopic examination was positive in 29 patients, fungal pathogens were isolated from 41 patients (31 Aspergillus, 8 Candida, 1 Fusarium, and 1 hyaline septate hyphae), beta-d-glucan was positive in 34 cases while galactomannan was positive in 45 cases. The isolated Aspergillus species were $11 \mathrm{~A}$. flavus, $11 \mathrm{~A}$. fumigatus, 4 A. niger, 2 A. nidulans, 1 A. terreus, and 2 Aspergillus spp.

\section{Sensitivity, specificity, PPV, and NPV}

Sensitivity, specificity, PPV, NPV, and total accuracy of individual tests were calculated according to EORTC/MSG categories; probable against unlikely IFI cases for IFI and for IPA as shown in Table 1.

Table 1: Sensitivity, specificity, PPV, NPV, and total accuracy of different tests considering EORTC/MSG categories for IFI and IPA in 104 patients with clinically suspected LRT fungal infection (probable against no IFI)

\begin{tabular}{llll}
\hline & Fungal culture & Galactomannan & BDG \\
& IFI (IPA) (\%) & IFI (IPA) (\%) & IFI (IPA) (\%) \\
\hline Sensitivity & $77.8(76.7)$ & $84.4(88.4)$ & $44.4(41.9)$ \\
Specificity & $85.4(85.4)$ & $82.9(82.9)$ & $70.7(70.7)$ \\
PPV & $85.4(84.6)$ & $84.4(84.4)$ & $62.5(60.0)$ \\
NPV & $77.8(77.8)$ & $82.9(87.2)$ & $53.7(53.7)$ \\
Total accuracy & $81.4(81.0)$ & $83.7(85.7)$ & $57.0(56.0)$ \\
\hline IFI. Invasive fungal infection, IPA: Invasive pulmonary aspergillosis.
\end{tabular}

\section{Combined tests}

When we compared different combinations of tests, fungal culture and galactomannan had the best sensitivity (100\%) and best specificity $(73.2 \%)$, followed by the combination of galactomannan with BDG, which had sensitivity (93.3\%) and specificity (65.9\%). Performance of different combinations of tests was calculated also according to EORTC/MSG categories, as shown in Table 2.

Table 2: Performance of different combinations of tests calculated according to EORTC/MSG categories (probable against no IFI)

\begin{tabular}{lllll}
\hline & Fungal/GM (\%) & Fungal/BDG (\%) & GM/BDG (\%) & Fungal/GM/BDG (\%) \\
\hline Sensitivity & 100 & 93.3 & 93.3 & 100 \\
Specificity & 73.2 & 63.4 & 65.9 & 63.4 \\
PPV & 80.4 & 73.7 & 75 & 75 \\
NPV & 100 & 89.7 & 90 & 100 \\
Total accuracy & 87.2 & 78.5 & 80.2 & 82.6 \\
\hline
\end{tabular}

Figures 2 and 3 demonstrate the different performances of GM and BDG in different groups of the study. A significant different performance of $\mathrm{GM} ; \mathrm{p}=$ 0.0008 , but not BDG; $p=0.121$, was detected in patients with malignant disorders when compared to non-malignant cases. Galactomannan had better performance among malignant patients, especially hematological malignancies.

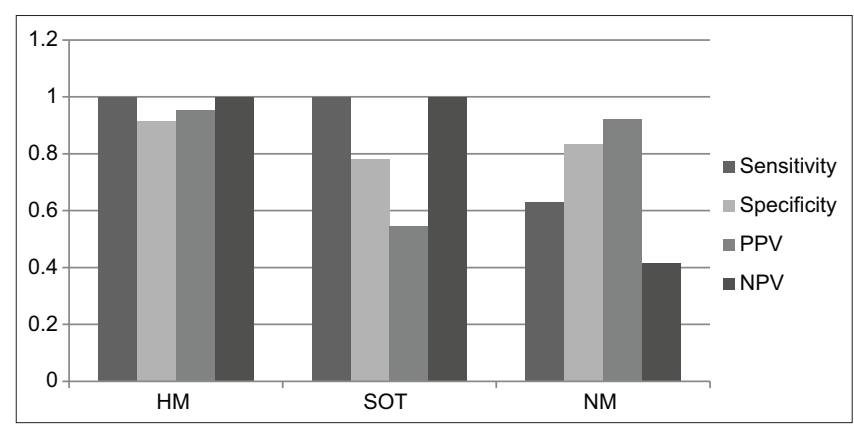

Figure 2: Performance of galactomannan among malignant and non-malignant patients. Hematological malignancies: Sensitivity, specificity, PPV, and NPV 100\%, 91.7\%, 95.2\%, and $100 \%$, respectively. Solid organ malignancies: Sensitivity, specificity, PPV, and NPV $100 \%, 78.3 \%, 54.5 \%$, and $100 \%$, respectively. Nonmalignant: Sensitivity, specificity, PPV, and NPV 63.2\%, 83.3\%, $92.3 \%$, and $41.7 \%$, respectively

\section{Discussion}

Invasive fungal infections of the respiratory tract are life threatening as they mainly occur in immunocompromised patients or those suffering from pre-existing lung diseases. Improving diagnostics is urgently required, especially in the sense of shorter time to result reporting. A negative result is as important as a positive result to avoid unnecessary treatment with its side effects on patients already receiving several medications and extra costs on the health system. Invasive fungal infections, particularly invasive aspergillosis (IA) is increasing among the high-risk population, mainly oncology, transplant recipients and patients with chronic obstructive pulmonary disease (COPD). Rapid and accurate diagnosis remains a big challenge because of non-specific clinical and radiological findings, low sensitivity, and delayed results of conventional culture. Therefore, non-culturebased methods, such as detection of DNA by PCR or measurements of fungal biomarkers in bronchoalveolar lavage (BAL) fluid, are important adjunctive tools [8].

Although fungal pathogens are known to cause disease in every system of the body, yet respiratory tract is the most common site of invasive fungal infections in high-risk patients. The serologic detection of BDG has been reported to be clinically useful and has been included in the EORTC/MSG criteria for the diagnosis of IFI but not yet validated for use in BAL specimens (2008). Therefore, we attempted to investigate the usefulness of the BDG 


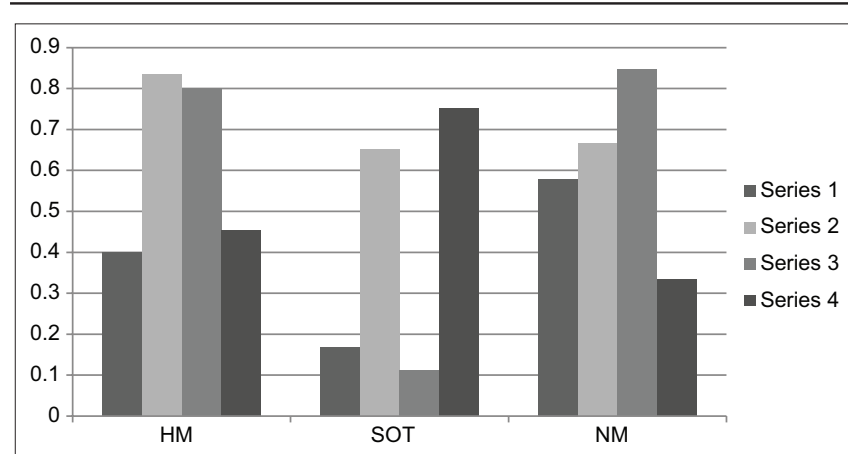

Figure 3: Performance of $\beta$-D-glucan in malignant and non-malignant patients. Hematological malignancies: Sensitivity, specificity, PPV, and NPV 40\%, 83.3\%, 80\%, and $45.5 \%$, respectively. Solid organ malignancies: Sensitivity, specificity, PPV, and NPV 16.7\%, 65.2\%, $11.1 \%$, and $75 \%$, respectively. Nonmalignant: Sensitivity, specificity, PPV, and NPV 57.9\%, 66.7\%, 84.6\%, and 33.3\%, respectively

test as compared to GM in BAL specimens in patients with suspected IFI of LRT.

Demonstration of fungal elements in biopsy tissue is necessary for a definitive diagnosis of invasive fungal infection (IFI). When a biopsy is not possible, positive respiratory culture (sputum or BAL fluid) is highly predictive of invasive disease in a high-risk patient. The use of galactomannan in serum as a diagnostic test for the detection of IFI is well established since it was added by the consensus of definitions IFI from EORTC/MSG to the laboratory criteria confirming IFI [6]. Galactomannan determination in BAL showed high sensitivity and specificity for aspergillosis in several studies [9] and [10]. The use of galactomannan in BAL is considered the gold standard method in the diagnosis of invasive aspergillosis [11].

In the present study, galactomannan showed reliable sensitivity and specificity $(84 \%$ and $83 \%$, respectively) at optic density index ODI of 1.0 for diagnosis of IFI and ( $88 \%$ and $83 \%$, respectively) for diagnosis of IPA; this is due to most of the cases were caused by Aspergillus. In several studies, on comparing the performance of galactomannan detection in BAL fluid to that of serum, better sensitivity was recorded when using BAL as a specimen, with a sensitivity, specificity, PPV, and NPV; $56 \%, 97 \%, 83 \%$, and $88 \%$ in BAL, respectively, and $33 \%, 97 \%, 100 \%$, and $83 \%$ in serum, respectively. Overall moderate sensitivity was recorded for galactomannan when used as a single biomarker [9]. Although galactomannan had repeatedly shown high specificity in BAL fluid, its performance as a single test was not fully satisfactory; besides its optimum cutoff was not agreed upon [12]. Some molds do not produce either galactomannan or BDG (e.g., Mucor and Rhizopus) and this means that a negative test does not rule out invasive fungal infection [13]. Combining fungal culture to galactomannan detection in BAL in the current study showed the best values for sensitivity, specificity, PPV, and NPV $(100 \%, 73.2 \%, 80.4 \%$, and $100 \%$, respectively). Still, the delayed results of culture compromise the use of this combination for routine rapid therapeutic decisions in high-risk patients. Hence, it is obvious that another biomarker is the optimum test required to improve the diagnostic performance of $\mathrm{GM}$ in BAL fluid to ensure rapid results.

In the present study, sensitivity and specificity, PPV, and NPV of BAL BDG were $44 \%, 71 \%, 62 \%$, and $54 \%$ when compared to $84 \%, 83 \%, 84 \%$, and $84 \%$ of BAL GM, respectively; the poor sensitivity and NPV of BAL BDG could hinder its use as a routine single test for the diagnosis of IFI. Similarly, it was concluded that the accuracy of BDG testing in BAL specimen is marginal and can only be used as a part of the full assessment with clinical and radiological findings for the diagnosis of invasive fungal infections [14]. It is thus evident that GM has a better performance in BAL than BDG. In a metaanalysis review including studies carried out on 6244 cases at risk of IFI, the accuracy of BDG test on blood samples varied widely with better performance in serum than BAL samples [15]. When studying BDG testing in BAL for all IFI, Rose et al. showed that it has a similar sensitivity to that of BAL GM (both $71 \%$ ), but with lower specificity ( $68 \%$ vs. $98 \%$, respectively) in the diagnosis of IFI in high-risk patients [16]. It was concluded that the poor specificity and low reproducibility of BAL BDG may limit its use in the clinical setting $[14,16]$.

Many factors affect the results of BDG in BAL fluid, mainly antifungal therapy at the time of bronchoscopy [16], [17]. Other factors affecting the sensitivity and specificity of this test could be Candida colonization, and the type of fungal pathogen causing the infection. The diagnostic performance of BAL BDG as a single test was less than that of blood in another meta-analysis; with better results on combining testing GM in BAL to BDG in serum samples [9]. Similarly, combining both tests increased their performance in the current study up to $90 \%$.

In the current study, there was a difference in the differential distribution of probable, possible and unlikely IFI cases among malignant and nonmalignant groups studied. More probable cases were encountered among non-malignant cases than patients with malignant disorders $(76 \%$ versus $33 \%$ respectively). Similarly, it was concluded that probable IFI was more commonly reported among patients with chronic obstructive pulmonary disease, constituting $76.3 \%$ (61/80), [18]. In the present study, combining the results of serological markers, namely GM and BDG, resulted in improved performance of BDG especially in hematological malignancy cases, but not in solid organ tumors and non-malignant cases with IFI. In their study, Mahmoud and colleagues (2019), serum BDG was positive in 12 of 61 cases (20\%) of COPD patients with probable pulmonary fungal infections [18]. In agreement with our results Lamoth, (2016), stated that a better sensitivity of BDG test was reported in patients with hematological malignancy when compared to solid organ transplant recipients or patients with less severe immunosuppression [19]. 


\section{Conclusion}

The present study does not support the use of BDG assay in BAL specimens for the diagnosis of IFI as a single test due to its poor performance. However, its combined use with fungal culture or GM in BAL improved its results, especially in patients with hematological malignancies. The type of host, as well as other factors, may influence the utility of biomarkers. Thus, we recommend limiting the use of BDG in BAL to highly suspicious patients with neutropenia and to couple its use to galactomannan results.

\section{References}

1. Bassetti M, Garnacho-Montero J, Calandra T, Kullberg B, Dimopoulos G, Azoulay E, et al. Intensive care medicine research agenda on invasive fungal infection in critically ill patients. Intensive Care Med. 2017;43(9):1225-38. https://doi. org/10.1007/s00134-017-4731-2

PMid:28255613

2. Patterson TF, Thompson GR, Denning DW, Fishman J, Hadley S, Herbrecht $R$, et al. Practice guidelines for the diagnosis and management of aspergillosis: 2016 update by the Infectious Diseases Society of America (IDSA). Clin Infect Dis. 2016;63(4):433-42. https://doi.org/10.1093/cid/ciw444 PMid:27365388

3. Chu YF. Oats Nutrition and Technology. Barrington, Illinois: Wiley Blackwell; 2014

4. Odabasi Z, Paetznick V, Rodriguez J, Chen E, McGinnis M, Ostrosky-Zeichner L. Differences in beta-glucan levels in culture supernatants of a variety of fungi. Med Mycol. 2006;44(3):26772. https://doi.org/10.1080/13693780500474327 PMid:16702107

5. Marty F, Koo S. Role of (1-->3)-beta-D-glucan in the diagnosis of invasive aspergillosis. Med Mycol. 2009;47(Suppl 1):S23340. https://doi.org/10.1080/13693780802308454

PMid:18720216

6. De Pauw B, Walsh TJ, Donnelly JP, Stevens D, Edwards J, Calandra $\mathrm{T}$, et al. Revised definitions of invasive fungal disease from the European Organization for Research and Treatment of Cancer/Invasive Fungal Infections Cooperative Group and the National Institute of Allergy and Infectious Diseases Mycoses Study Group (EORTC/MSG) Consensus Group. Clin Infect Dis. 2008;46(12):1813-21. https://doi.org/10.3410/f.1116756.572830 PMid:18462102

7. El-Mahallawy H, Shaker H, Helmy H, Mostafa T, Abo-Sedah A. Evaluation of pan-fungal PCR assay and Aspergillus antigen detection in the diagnosis of invasive fungal infections in high risk paediatric cancer patients. Med Mycol. 2006;44(8):733-9. https://doi.org/10.1080/13693780600939955

PMid:17127630

8. Park S, Yoon J, Kim SH. Voriconazole-refractory invasive aspergillosis. Korean J Intern Med. 2017;32(5):805-12. https:// doi.org/10.3904/kjim.2017.109

PMid:28835093
9. Boch T, Spiess B, Cornely OA, Vehreschild JJ, Rath PM, Steinmann $\mathrm{J}$, et al. Diagnosis of invasive fungal infections in haematological patients by combined use of galactomannan, 1,3- $\beta$-D-glucan, Aspergillus PCR, multifungal DNA-microarray, and Aspergillus azole resistance PCRs in blood and bronchoalveolar lavage samples: Results of a prospective multicentre study. Clin Microbiol Infect. 2016;22(10):862-8. https://doi.org/10.1016/j.cmi.2016.06.021

PMid:27393123

10. Zou M, Tang L, Zhao Z, Chen L, Chen P, Huang Z, et al. Systematic review and meta-analysis of detecting galactomannan in bronchoalveolar lavage fluid for diagnosing invasive aspergillosis. PLoS One. 2012;7(8):e43347. https://doi. org/10.1371/journal.pone.0043347 PMid:22905261

11. Hoenigl M, Prattes J, Spiess B, Wagner J, Prueller F, Raggam R, et al. Performance of galactomannan, beta-D-glucan, Aspergillus lateral-flow device, conventional culture, and PCR tests with bronchoalveolar lavage fluid for diagnosis of invasive pulmonary aspergillosis. J Clin Microbiol. 2014;52(6):2039-45. https://doi.org/10.1128/jcm.00467-14

PMid:24671798

12. Cefalo M, Puxeddu E, Samati L, Paterno G, Fontana C, Nasso D, et al. Diagnostic performance and safety of bronchoalveolar lavage in thrombocytopenic haematological patients for invasive fungal infections diagnosis: A monocentric, retrospective experience. Mediterr J Hematol Infect Dis. 2019;11(1):e2019065. https://doi.org/10.4084/mjhid.2019.065 PMid:31700590

13. Pichun MB, Raed S, Gea-Banacloche JC. Infectious complications in oncology. In: Abraham J, Gulley JL, Allegra CJ, Pine JW, editors. The Bethesda Handbook of Clinical Oncology. Clevland: Lippincott Williams and Wilkins; 2014. p. 448-67.

14. Shi $X Y$, Liu $Y$, Gu XM, Hao SY, Wang YH, Jiang SJ. Diagnostic value of $(1 \rightarrow 3)-\beta$-D-glucan in bronchoalveolar lavage fluid for invasive fungal disease: A meta-analysis. Respir Med. 2016;117:48-53. https://doi.org/10.1016/j.rmed.2016.05.017 PMid:27492513

15. White SK, Schmidt RL, Walker BS, Hanson KE. $(1 \rightarrow 3)-\beta-D$ glucan testing for the detection of invasive fungal infections in immunocompromised or critically ill people. Cochrane Database Syst Rev. 2020;7(7):CD009833. https://doi. org/10.1002/14651858.cd009833.pub2

PMid:32693433

16. Rose SR, Vallabhajosyula S, Velez MG, Fedorko D, VanRaden M Gea-Banacloche J, et al. The utility of bronchoalveolar lavage beta-d-glucan testing for the diagnosis of invasive fungal infection. J Infect. 2014;69(3):278-83. https://doi.org/10.1016/j. jinf.2014.04.008

PMid:24797077

17. Prattes J, Flick H, Pruller F, Koidl C, Raggam R, Palfner M, et al. Novel tests for diagnosis of invasive aspergillosis in patients with underlying respiratory diseases. Am J Respir Crit Care Med. 2014;190(8):922-9. https://doi.org/10.1164/ rccm.201407-1275oc PMid:25203869

18. Mahmoud E, El-Din MM, Hafez M, Sobh E, Ibrahim R. Pulmonary fungal infection in patients with acute exacerbation of chronic obstructive pulmonary disease. Sci J Al Azhar Med Fac Girl. 2019;3:7-13. https://doi.org/10.4103/sjamf.sjamf_37_18

19. Lamoth F. Galactomannan and 1,3- $\beta$-D-glucan Testing for the diagnosis of invasive aspergillosis. J Fungi (Basel). 2016;2(3):22. https://doi.org/10.3390/jof2030022 PMid:29376937 